\title{
Assessment of decorin-binding protein A to the infectivity of Borrelia burgdorferi in the murine models of needle and tick infection Jon S Blevins, Kayla E Hagman and Michael V Norgard*
}

\author{
Address: Department of Microbiology, University of Texas Southwestern Medical Center, Dallas, Texas 75390, USA \\ Email: Jon S Blevins - jon.blevins@utsouthwestern.edu; Kayla E Hagman -yogablonde@yahoo.com; \\ Michael V Norgard* - michael.norgard@utsouthwestern.edu \\ * Corresponding author
}

Published: 28 May 2008

BMC Microbiology 2008, 8:82 doi:10.1/86/|47|-2180-8-82

Received: I February 2008

Accepted: 28 May 2008

This article is available from: http://www.biomedcentral.com/I47I-2/80/8/82

(c) 2008 Blevins et al; licensee BioMed Central Ltd.

This is an Open Access article distributed under the terms of the Creative Commons Attribution License (http://creativecommons.org/licenses/by/2.0), which permits unrestricted use, distribution, and reproduction in any medium, provided the original work is properly cited.

\begin{abstract}
Background: Decorin-binding proteins (Dbps) A and B of Borrelia burgdorferi, the agent of Lyme disease, are surface-exposed lipoproteins that presumably bind to the extracellular matrix proteoglycan, decorin. B. burgdorferi infects various tissues including the bladder, heart, joints, skin and the central nervous system, and the ability of B. burgdorferi to bind decorin has been hypothesized to be important for this disseminatory pathogenic strategy.
\end{abstract}

Results: To determine the role of DbpBA in the infectious lifecycle of $B$. burgdorferi, we created a DbpBA-deficient mutant of $B$. burgdorferi strain 297 and compared the infectious phenotype of the mutant to the wild-type strain in the experimental murine model of Lyme borreliosis. The mutant strain exhibited a 4-log decrease in infectivity, relative to the wild-type strain, when needle inoculated into mice. Upon complementation of the DbpBA-mutant strain with DbpA, the wildtype level of infectivity was restored. In addition, we demonstrated that the DbpBA-deficient mutant was able to colonize lxodes scapularis larval ticks after feeding on infected mice and persist within the ticks during the molt to the nymphal state. Moreover, surprisingly, the DbpBA-mutant strain was capable of being transmitted to naïve mice via tick bite, giving rise to infected mice.

Conclusion: These results suggest that DbpBA is not required for the natural tick-transmission process to mammals, despite inferences from needle-inoculation experiments implying a requirement for $\mathrm{DbpBA}$ during mammalian infection. The combined findings also send a cautionary note regarding how results from needle-inoculation experiments with mice should be interpreted.

\section{Background}

The causative agent of Lyme disease, Borrelia burgdorferi, is introduced into a mammalian host via tick bite, whereupon the organisms enter the skin, disseminate hematogenously, and persist in the presence of a strong host immune response [1]. The dissemination and persistence of $B$. burgdorferi within a mammalian host is thought to be predicated, at least in part, on the organism's ability to bind molecules of the extracellular matrix (ECM) [2-4], inasmuch as these interactions have been shown to be important for other bacterial pathogens [5]. Among various ECM components, $B$. burgdorferi binds to type I collagen [4], fibronectin $[6,7]$, integrins $[8,9]$, the proteoglycan decorin [10], and glycosaminoglycans (GAGs) $[11,12]$. The $B$. burgdorferi proteins described as ECM-binding proteins include BBK32 [6,13], Bgp (borrelia-GAG binding protein) [12], P66 (Oms66) [14], decorin-binding protein (Dbp) A, and DbpB $[10,15]$. These proteins, and perhaps 
other as yet unidentified molecules, may play a significant role in the infectivity and pathogenesis phenotypes of $B$. burgdorferi.

Our laboratory has been interested in DbpA as a vaccine candidate for the prevention of Lyme disease and the contribution of DbpA and DbpB to B. burgdorferi pathogenesis and infectivity [16,17]. Since their in vitro characterization as decorin-binding proteins $[10,15]$, the DbpA and DbpB lipoproteins have been implicated as potential contributors to adhesion and colonization of $B$. burgdorferi within mammalian hosts $[15,18]$. The genes that encode DbpA and DbpB reside in an operon, $d b p B A$, on linear plasmid 54 (lp54) and are found within many B. burgdorferi sensu lato isolates [19]. Neither protein is expressed by $B$. burgdorferi within the tick vector [17], however, expression of $d b p A$ (and presumably $d b p B$ ) is upregulated in the mammalian host after ticks deposit spirochetes into the skin $[20,21]$. DbpA and DbpB expression likely remains high for the duration of mammalian infection, as inferred by the presence of antibodies against both antigens in the serum of mice as late as one year after infection (Hagman, unpublished data). The presence of antibodies against DbpA in the serum of patients with late-stage, disseminated Lyme disease also is well-documented [22], providing added support for DbpA expression by $B$. burgdorferi during chronic infection.

Although the combined data to date suggest an important role for the decorin-binding proteins of $B$. burgdorferi during mammalian infectivity, virtually all data inferring the importance of DbpA and DbpB thus far have been indirect. The first direct investigation into the role of the $d b p B A$ operon in the infectious lifecycle of B. burgdorferi was carried out by Shi et al [23]. In this study, mutational analysis of $d b p B A$ in $B$. burgdorferi strain $\mathrm{B} 31$ indicated that neither DbpA, nor DbpB was essential in the murine needle-challenge infection model of borreliosis. However, there was evidence suggesting that these mutants exhibited a modest level of attenuation in immunocompetent mice. Unfortunately, a comprehensive $50 \%$ infectious dose $\left(\mathrm{ID}_{50}\right)$ was not included in this report to further investigate this possible defect, nor was genetic complementation of the mutation performed. This latter point is of particular importance given the genetic plasticity of $B$. burgdorferi and the possibility that spontaneous loss of an endogenous borrelial plasmid might account for the apparent defect in this mutant. To more directly examine the role of both DbpA and DbpB in the murine/tick model of Lyme disease, a $d b p B A$-deficient mutant and a $d b p A$ genetic complement of the mutant were generated in the infectious strain 297 of B. burgdorferi. Phenotypic assessment of mouse needle infectivity by $\mathrm{ID}_{50}$ analysis and Ixodes scapularis tick colonization and tick-transmis- sion capacity by the $\mathrm{Bb} 297 d b p B A$-mutant also were performed.

\section{Results \\ Construction and characterization of a dbpBA-deficient mutant}

To assess the roles that DbpA or DbpB play in the infectivity and pathogenesis of $B$. burgdorferi, we created a $d b p B A$ deficient mutant by allelic exchange of a PflgB-kan cassette for the majority of the $d b p B A$ operon (Fig. 1A). Infectious Bb297 was chosen for construction of the $d b p B A$ mutant because it is a human isolate [24] and prior infectious mutants of this strain have been readily created [25-28].

$\mathrm{Bb} 297$ was electroporated with the suicide vector, $\mathrm{pKHd}$ bpAko, containing a PflgB-kan cassette flanked by $1.5 \mathrm{~kb}$ of DNA on the left side of the $d b p B A$ operon and $890 \mathrm{bp}$ of DNA on the right side of the $d b p B A$ operon (Fig. 1A). This allelic exchange strategy relies on a double crossover, homologous recombination event to replace the $d b p B A$ operon with the PflgB-kan cassette. Several kanamycinresistant transformants were obtained, and the presence (Fig. 1B) and orientation (Fig. 1C) of the PflgB-kan cassette within the $d b p B A$ operon was confirmed by PCR analysis.

During genetic manipulation and in vitro cultivation, $B$. burgdorferi may spontaneously lose endogenous plasmids that are not required for growth in vitro, but are essential for mammalian infection. At least two plasmid-encoded genes, vlsE [29] and pncA (BBE22) [30], fit this criteria. One kanamycin-resistant transformant, BbKH500, retained both $v l s E$ and $p n c A$ (Fig. 2) and was analyzed by immunoblotting to confirm the loss of DbpA and DbpB. As expected, DbpA and DbpB were absent from BbKH500 (Figs. 3A and 3B). BbKH500 exhibited identical doubling times when compared to wild-type Bb297 (data not shown) and PCR-based plasmid profiling revealed that the endogenous plasmid profile of BbKH500 matched that of the parent Bb297 (Fig. 2).

\section{Loss of DbpBA significantly reduces the infectivity of $B$. burgdorferi in mice when introduced via needle inoculation}

To determine the contribution of $\mathrm{DbpA}$ and $\mathrm{DbpB}$ to the infectivity of $B$. burgdorferi in mice, $\mathrm{C} 3 \mathrm{H} / \mathrm{HeJ}$ mice were challenged intradermally via needle inoculation with increasing numbers of BbKH500 $\left(10^{4}, 10^{5}, 10^{6}\right.$ and $10^{7}$ spirochetes). As a positive control, an additional five mice were inoculated with wild-type Bb297 at a dose of $10^{3}$ spirochetes ( $\mathrm{ID}_{50}$ of approximately 50 spirochetes; [27]). The results are shown in Table 1 . Whereas the mice infected with $10^{3}$ of Bb297 showed culture positive ear punch biopsies at two weeks post-infection, needle challenge of naïve mice with $10^{4} \mathrm{BbKH} 500$ did not produce an infec- 

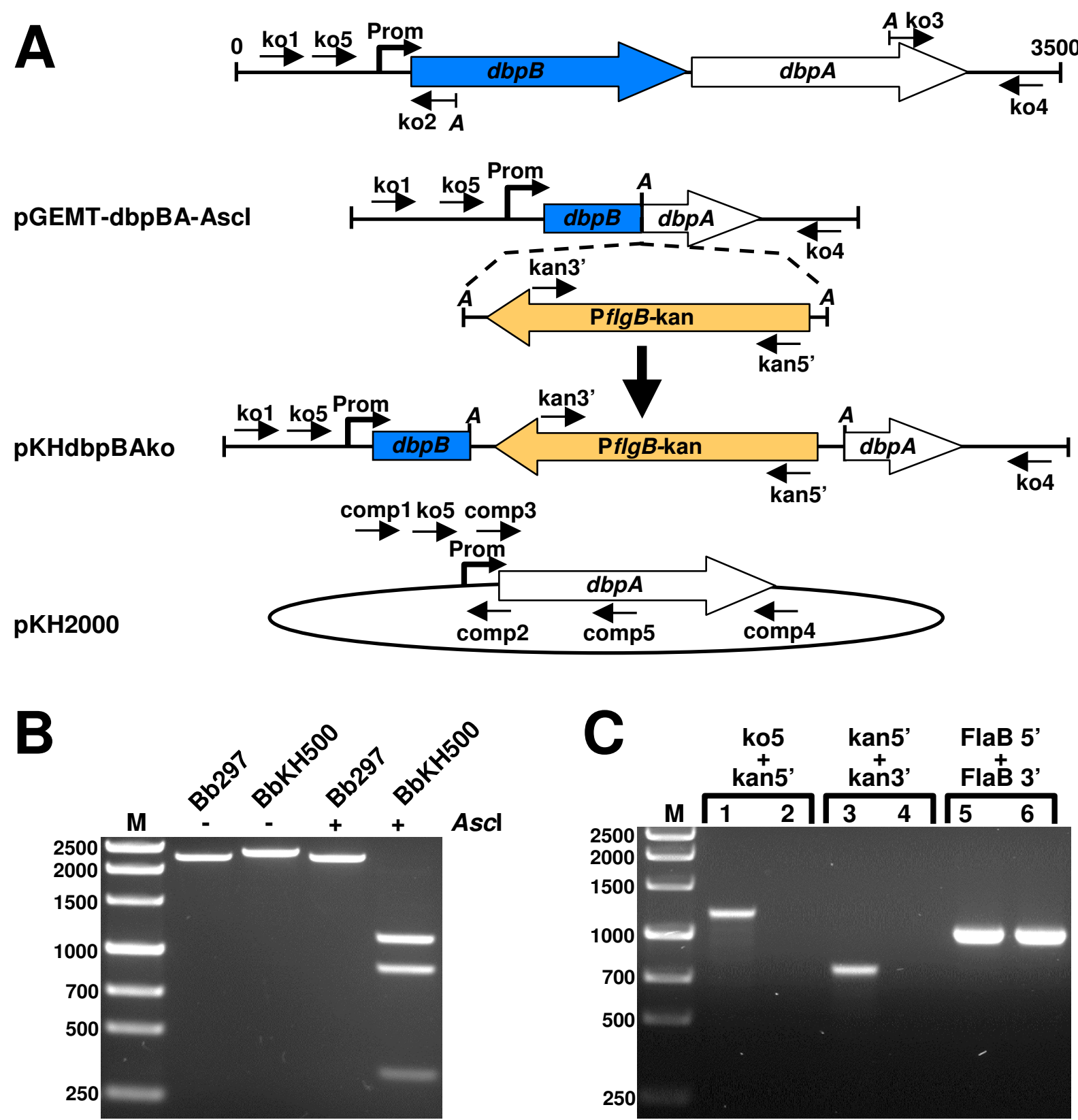

Figure I

Construction of dbpBA-deletion mutant BbKH500 and Prom-dbpA complementation vector and PCR confirmation. (A) Strategy for the replacement of the dbpBA operon with the PflgB-kan and complementation with $\mathrm{pKH} 2000$. $\mathrm{PKH}$ dbpBAko was the pGEM-T easy-based suicide plasmid used to transform Bb297 for the homologous recombination of the kanamycin-resistance gene into the $\mathrm{dbpBA}$ operon. "A" denotes Ascl sites. PflgB-kan denotes the kanamycin-resistance marker expressed from the flgB promoter. The borrelial shuttle vector containing the $\mathrm{dbpBA}$ Prom fused to the $\mathrm{dbpA} O \mathrm{ORF}$ ( $\mathrm{pKH} 2000$ ) was transformed into BbKH500 to restore DbpA expression. Oligonucleotide primers used for PCR are indicated with short arrows. (B) PCR using primers ko5 and ko4 (shown in panel A). The first two lanes are undigested PCR products from Bb297 and BbKH500, whereas the second two lanes are the corresponding PCR products digested with AsCl. (C) Lanes I, 3 and 5 are PCR products derived from BbKH500 template DNA and lanes 2, 4 and 6 are PCR products derived from Bb297 template DNA. Primer pairs used in PCR are indicated above the lanes. FlaB5' and FlaB3' primers amplify flaB of B. burgdorferi. DNA size standards (M) are shown in base pairs on the left. 


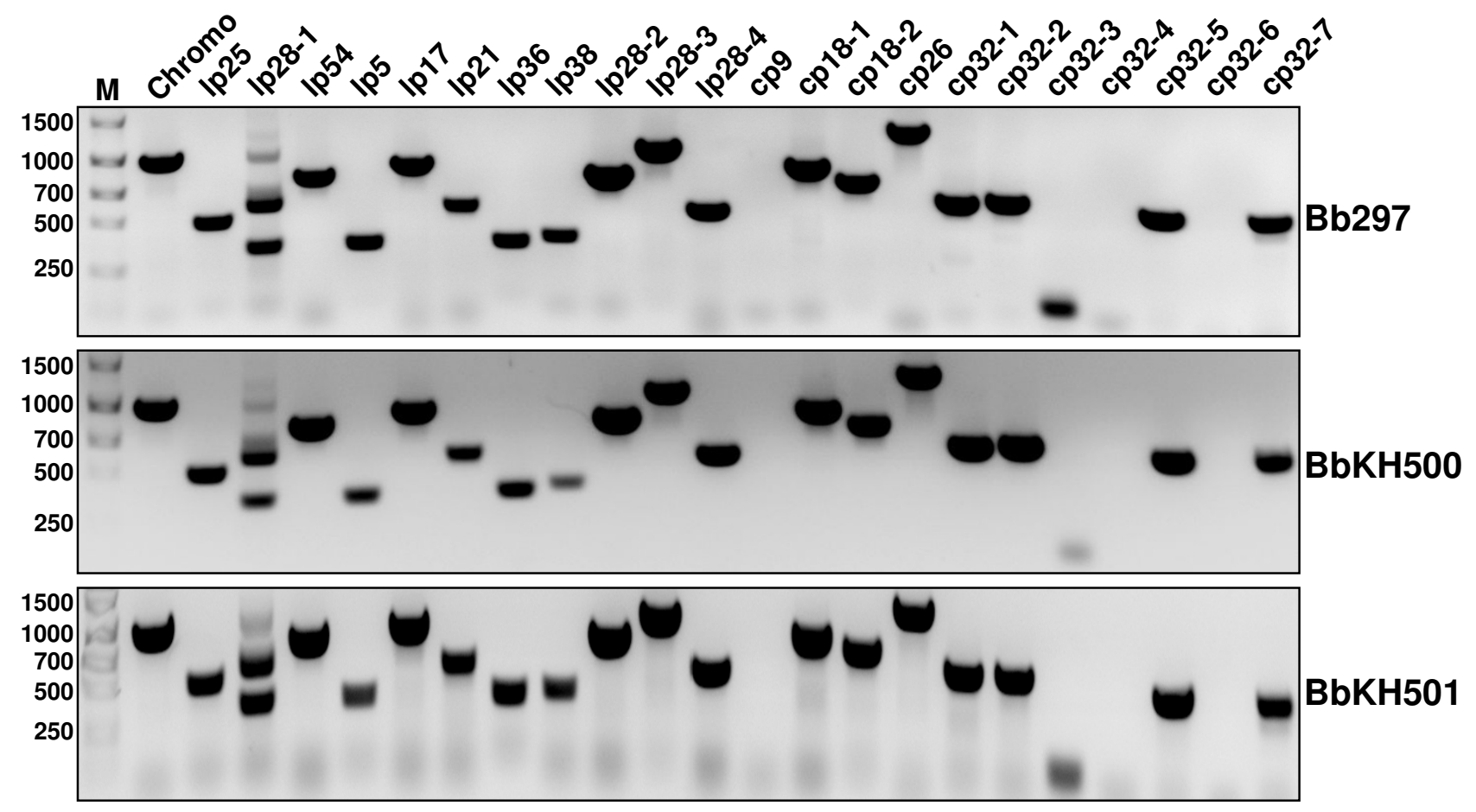

Figure 2

PCR-based plasmid profiling to compare the plasmid contents of the strains employed in this study. PCR amplification with primers specific for each of the known endogenous $B$. burgdorferi plasmids was used to compare the plasmid content of parent $\mathrm{Bb} 297$, BbKH500, and BbKH50I. Sequence information for the primers utilized is provided in Table 4. Plasmid designations above each lane are based on strain B3I plasmid annotation. DNA size standards (M) are shown in base pairs.

tion in any mouse $(\mathrm{n}=15)$, even when the infection was allowed to progress for 14 weeks. Seventeen mice challenged with $10^{5}$ and ten mice challenged with $10^{6}$ BbKH500 also did not show signs of infection (negative ear-punch biopsy culture) at four weeks post-challenge (Table 1). However, after 10 weeks, three mice from the group infected with $10^{5} \mathrm{BbKH} 500$ and two mice from the group challenged with $10^{6} \mathrm{BbKH} 500$ were shown to be infected by positive ear-punch biopsy cultures. Additionally, mice were challenged with either $10^{5}$ or $10^{6}$ BbKH500 and ear-punch biopsies were harvested 14 weeks after infection (Table 1). From the group that received $10^{5}$ spirochetes, two mice were infected $(n=10)$, and from the group that received $10^{6}$ spirochetes, four mice were infected $(n=10)$. All of the mice challenged with $10^{7}$ of BbKH500 had positive ear-punch cultures as early as five-weeks post-challenge, and as late as 14 weeks post-infection $(n=5)$. These spirochetes from ear punchpositive cultures were analyzed by diagnostic PCR as described above, and confirmed to be Bb297 or BbKH500 (data not shown). Aliquots of these cultures also were passed to media containing kanamycin to confirm resistance and sensitivity of BbKH500 and Bb297, respectively. Seroconversion analyses performed on a subset of mice also revealed that none of the mice infected with BbKH500 showed antibody reactivity against DbpA or DbpB (data not shown). Furthermore, only mice that exhibited culture-positive ear punch cultures showed significant serum antibody reactivity with P39 (data not shown). Analysis of all infection results yielded an $\mathrm{ID}_{50}$ for BbKH500 of $1.2 \times 10^{6}(\mathrm{p}<0.001)$ compared with $\mathrm{ID}_{50}$ of approximately 50 bacteria for wild-type $\mathrm{Bb} 297$ [27].

\section{Complementation of the dbpBA-deletion mutant with DbpA restores infectivity in needle-challenged mice} The results above suggested that the $d b p B A$ operon is required for full infectivity of $B$. burgdorferi when mice are infected via needle inoculation. However, during genetic manipulation of $B$. burgdorferi, it is not uncommon to lose one or more plasmid(s) which potentially contribute to infection, therefore genetic complementation is necessary to definitely ascribe the attenuated phenotype in BbKH500 to the $d b p B A$ lesion. Although BbKH500 carries a mutation in both DbpA and DbpB, we chose to complement with only $d b p A$ because i) DbpA is better characterized that DbpB $[31,32]$ and ii) experimentation suggests that DbpA is the prominent Dbp in B. burgdorferi $[15,33]$. To restore DbpA expression in BbKH500, a shuttle plas- 
Table I: Assessing infectivity of BbKH500 in needle-challenged $\mathrm{C} 3 \mathrm{H} / \mathrm{HeJ}$ mice.

\begin{tabular}{cllll}
\hline & \multicolumn{4}{c}{ Inoculation dose (bacteria/inoculation) } \\
\cline { 2 - 5 } Time post-infection (wks) & $10^{4 a}$ & $10^{5 a}$ & $10^{6 a}$ & $10^{7 a}$ \\
\hline 5 & $0 / 10$ & $0 / 17$ & $0 / 10$ & $5 / 5$ \\
10 & ND & $3 / 17$ & $2 / 10$ & ND \\
$14 \mathrm{~b}$ & $0 / 5$ & $2 / 10$ & $4 / 10$ & $5 / 5$ \\
Cumulative results & $0 / 15$ & $5 / 27$ & $6 / 20$ & $10 / 10$
\end{tabular}

a Mice were needle infected with varying concentrations of bacteria. Infection rates were assessed at the noted timepoints by culturing $\mathrm{BbKH} 500$ from ear-punch biopsies.

b Mice from 14 week timepoint represent groups distinct from the 5and 10 -week groups.

mid carrying a copy of $d b p A$ driven by its native promoter was constructed (Fig. 1A). Since $d b p A$ is the second gene in the $d b p B A$ operon, we chose to clone the promoter for this operon upstream of the $d b p A$ gene and then insert the fusion product in the multiple-cloning site of pJD51. BbKH500 cells were transformed with this shuttle plasmid (pKH2000), yielding clones resistance to both kanamycin and streptomycin. These transformants were screened by PCR to verify the presence of the complementing plasmid (Fig. 3C). Clones shown to contain the complementing plasmid were checked for $v l s E$ and $p n c A$ (Fig 2.), as described above. PCR-based plasmid profiling, performed on one of the transformants that contained both $p n c A$ and $v l s E$, revealed a profile identical to the parent strain, BbKH500 (Fig. 2). This clone, designated BbKH501, was selected for further analysis and DbpA expression was confirmed by both SDS-PAGE (Fig. 3A) and immunoblot analysis (Fig. 3B). Proteinase $\mathrm{K}$ digestion of BbKH501 and Bb297, under conditions that left FlaB intact, demonstrated surface exposure of DbpA in both strains (Fig. 3D). Three groups of five $\mathrm{C} 3 \mathrm{H} / \mathrm{HeJ}$ mice were needle inoculated (i.d.) with BbKH501 at 102, $10^{3}$ or $10^{4}$ borreliae per mouse. Ear punch biopsies were harvested at either two weeks $\left(10^{3}\right.$ and $10^{4}$ doses $)$ or three weeks ( $10^{2}$ dose); all 15 mice became infected, as confirmed by positive ear-punch biopsy cultures. Although a precise determination of the $\mathrm{ID}_{50}$ of BbKH501 could not be calculated, the fact the $100 \%$ of the mice were infected with $10^{2}$ bacteria suggests that the $\mathrm{ID}_{50}$ is less than $10^{2}$ spirochetes (4-logs lower than BbKH500). Aliquots of these cultures were passed to media containing streptomycin and kanamycin selection to confirm resistance. Diagnostic PCR also confirmed that the spirochetes growing out of the ear punch biopsy were BbKH501 (data not shown). Seroconversion analyses carried out on a subset of mice also revealed that the mice infected with BbKH501 showed antibody reactivity against DbpA, but not DbpB
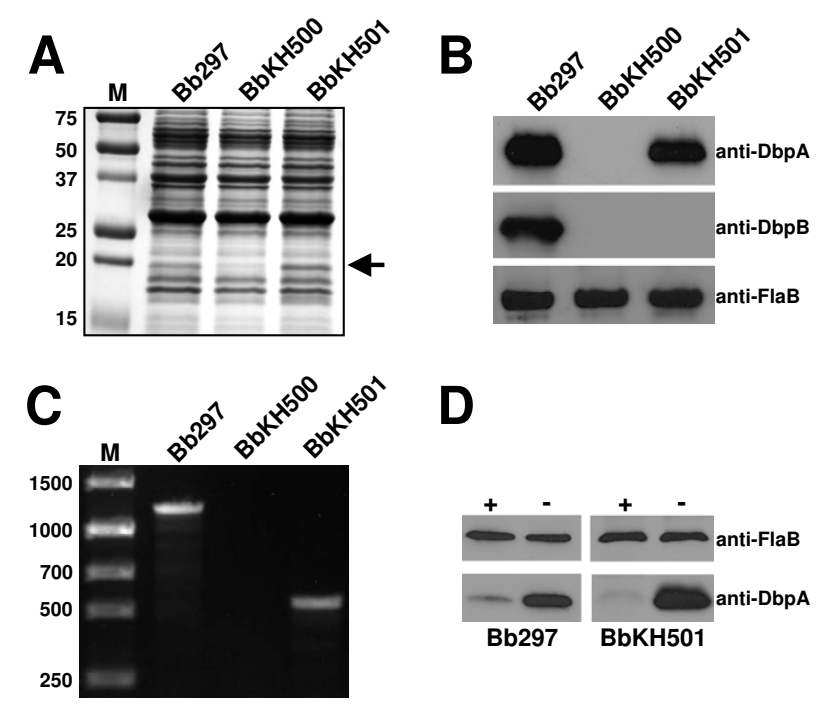

Figure 3

Characterization of BbKH500 and BbKH5OI. (A)

Whole-cell lysates of $\mathrm{Bb} 297, \mathrm{BbKH} 500$, and $\mathrm{BbKH} 50 \mathrm{I}$ were resolved by SDS-PAGE ( $12.5 \%$ acrylamide) and stained with Coomassie brilliant blue or (B) or transferred to nitrocellulose and assessed by immunoblot analysis with antibodies as noted on the right. (C) PCR analysis of Bb297, BbKH500 and BbKH50I for presence of dbpA. (D) Immunoblot analysis of whole-cell lysates of proteinase K-digested $(+)$ or undigested $(-) \mathrm{Bb} 297$ or BbKH50I. Antibodies used noted on the right. Molecular mass of markers (M) in panel $A$ are shown in $\mathrm{kDa}$. The arrow at the right in panel $A$ denotes the protein band corresponding to DbpA. DNA size standards $(M)$ in panel $C$ are shown in base pairs.

(data not shown). These data confirm that DbpA is necessary for wild-type levels of $B$. burgdorferi infectivity in mice when introduced via needle inoculation, and that $\mathrm{DbpB}$ is dispensable for this aspect of the infectious process.

\section{Neither DbpA nor DbpB is required for acquisition of $B$. burgdorferi by ticks or infection of mice via tick bite}

To determine whether I. scapularis ticks could acquire borreliae lacking $d b p A$ and $d b p B$, naïve larvae were allowed to feed to repletion on mice infected with BbKH500 or on mice infected with Bb297. Larvae were collected after feeding, allowed to molt, and the unfed nymphs were examined for the presence of $B$. burgdorferi by IFA; approximately 11 weeks had elapsed since these ticks had fed to repletion as larvae. Microscopic examination of multiple fields revealed approximately one spirochete per field in the BbKH500-infected ticks (23 ticks examined), as opposed to 20-30 spirochetes per field in the Bb297infected ticks (10 ticks examined). All Bb297-infected ticks examined were positive for $B$. burgdorferi. Of the ticks that had fed on BbKH500-infected mice, $80 \%$ were positive for spirochetes. 
Table 2: Assessing infectivity of BbKH500 via tick inoculation of $\mathrm{C} 3 \mathrm{H} / \mathrm{HeJ}$ mice

\begin{tabular}{lcc}
\hline Strain & Ticks/mouse $^{\mathrm{a}}$ & Mouse infectivity $^{\mathrm{b}}$ \\
\hline $\mathrm{Bb} 297$ & 5 & $2 / 2$ \\
$\mathrm{BbKH} 500$ & 5 & $1 / 2$ \\
& 10 & $4 / 6$ \\
\hline
\end{tabular}

a Varying number of ticks infected with BbKH500 were allowed to feed to repletion on naïve mice.

$\mathrm{b}$ Infection rates were assessed at three weeks post-infestation by culturing $\mathrm{BbKH} 500$ from ear-punch biopsies

Because the ticks maintained strain BbKH500 through their molt, it was important to assess whether the loss of DbpA and DbpB affected B. burgdorferi transmission to mice via tick bite. To test this, five BbKH500-infected nymphs were placed on each of two naïve mice and ten BbKH500-infected nymphs were placed on each of six naïve mice. An additional two naïve mice received five Bb297-infected ticks each. The ticks were allowed to feed to repletion, and at three weeks post-infestation, earpunch biopsies were harvested from all mice. The earpunch biopsies were cultured in BSK medium and the cultures examined by dark-field microscopy for the presence of B. burgdorferi. These results are shown in Table 2. One of the two mice infested with five ticks harboring BbKH500 was infected as demonstrated by a positive earpunch culture, and four of the six mice infested with ten ticks harboring BbKH500 were infected. Both of the mice on which the Bb297-infected ticks fed had positive earpunch biopsy cultures. These spirochetes were analyzed by diagnostic PCR as described above, and confirmed to be Bb297 or BbKH500 (data not shown). Seroconversion analyses performed on a subset of the BbKH500-infected mice also revealed that none of the mice infected with BbKH500 showed antibody reactivity against DbpA or DbpB (data not shown). Furthermore, only mice that exhibited culture-positive ear punch cultures showed significant antibody reactivity against P39 (data not shown). Taken together, these data indicate that DbpA and DbpB are not essential for the transmission of B. burgdorferi from ticks to mice.

\section{Discussion}

The importance of ECM-binding proteins to the pathogenic strategy of $B$. burgdorferi is not currently known. It is presumed that borreliae bind mammalian host cells during infection because binding to various ECM molecules and to tissue culture cells has been demonstrated in vitro $[4,34,35]$. Of the known ECM-binding proteins of B. burgdorferi, Bgp [36] and BBK32 [37] have been deleted from infectious strains $\mathrm{N} 40$ and B31, respectively, and shown to be dispensable for infectivity in the mouse model of Lyme disease. A deletion of integrin-binding protein, P66, in a noninfectious strain of B. burgdorferi, HB19 [38], caused the spirochetes to lose their ability to bind $b_{3}$ chain integrins [14] but the phenotype of a P66 mutant in an infectious strain of $B$. burgdorferi is currently unknown. The remaining two known ECM-binding proteins, DbpA and $\mathrm{DbpB}$, were the focus of this work due to multiple lines of evidence suggesting a role for one or both proteins during infection of mammalian hosts [15,18,31]. To directly address the contribution of both $\mathrm{DbpA}$ and $\mathrm{DbpB}$ to $B$. burgdorferi infectivity, we created BbKH500, a $d b p B A-$ deletion mutant of the human isolate $\mathrm{Bb} 297$, and used this strain to challenge mice with increasing numbers of spirochetes. We observed a 4-log reduction in infectivity of the mutant strain when the spirochetes were needle inoculated into mice. Complementation of the $d b p B A$ mutant with DbpA alone in the $d b p B A$-mutant restored infectivity of $B$. burgdorferi to wild-type levels.

Prior to this work, Fischer et al. [18] had restored DbpA and DbpB expression (via shuttle plasmid) to B. burgdorferi strain B314, a non-adherent, noninfectious derivative of strain B31 that has lost several plasmids, including lp54 [39]. Although expression of the Dbp molecules restored binding of the spirochetes to purified decorin, dermatan sulphate, and human epithelial cells [18], experiments to test the infectivity of B314 expressing DbpA and DbpB in the murine model of Lyme disease were implausible due to the loss of plasmids that are required for mammalian infection [39]. A recent study by Shi et al. [23] assessed the infectivity of a DbpA,B mutant of B. burgdorferi strain 5A18NP1, a B31 derivative that lacks the BBE02 gene [40]. Based on needle-challenge results obtained from a single inoculation dose of $10^{5}$ bacteria ( $80 \%$ infection), Shi et al. [23] ascertained that the $d b p B A$ locus was not required for infectivity. However, in the present study, we observed a 4-log increase in the $\mathrm{ID}_{50}$ of BbKH500 (>106 bacteria) and a $19 \%$ infection rate with a dose of $10^{5}$ BbHK500 when mice were infected by needle inoculation. The reason for the difference in infectivity levels of the $d b p B A$-deficient mutants reported by Shi et al. and BbKH500 created in our laboratory is unknown at this time. One possible explanation for this disparity could be due to our use of Bb297 as the parental strain for the present mutational analysis, whereas Shi et al. [23] utilized a clonal derivative of B31 that is a BBE02-mutant. This variability also could be explained by spirochete enumeration differences prior to needle challenge. As mentioned in the Methods, significant attention was given to the enumeration of BbKH500 prior to needle inoculation of mice.

It should be noted that during the course of the study described herein, a subsequent report on the role of DbpBA was published by Shi et al. [41], which, unlike their previous study, included genetic complementation experiments. Our current findings agree with those of this most recent report in that Shi et al. also observed a 4-log 
increase in $\mathrm{ID}_{50}$ values with their $d b p B A$ mutant [41]. In addition, Shi et al. observed a defect in the ability of the $d b p B A$ mutant to colonize heart, joint and skin tissues, suggesting an overall deficiency in dissemination [41]. The observation that mice infected with BbKH50O showed a delay in infectivity (10- to 14 -weeks post-infection) also suggests that BbKH500 might be attenuated with respect to its capacity to disseminate through the host. However, whereas we were able to compensate fully for the loss infectivity in the $d b p B A$ mutant by complementing with $d b p A$ alone (as assessed by ear punch biopsy culture), Shi et al. reported that both $d b p A$ and $d b p B$ were required to restore the infectivity of their $d b p B A$ mutant [41]. Although the precise reason for this disparity is unknown at this time, there are several differences between the experimental approaches of these studies that might account for these differing results. First, it is possible that some intrinsic differences(s) in the bacterial strains utilized might account for the variation observed between these two studies. Specifically, as noted above, strain 297 was the parental strain used in the current study, and Shi et al. utilized a highly-transformable clone (5A13) of strain B31 that lacks both lp56 and the virulence-associated plasmid lp25 as the background for their mutagenesis experiments $[30,41]$. Second, the disparity in our results might be due to the use of different strains of mice in these two studies; $\mathrm{C} 3 \mathrm{H} / \mathrm{HeJ}$ mice were used in our study, whereas Shi et al used BALB/C [41]. This may be relevant because numerous studies have reported that experimental infection of $\mathrm{C} 3 \mathrm{H}$ strains of mice with $B$. burgdorferi results in significantly different disease pathologies [42,43], higher spirochetal loads in multiple tissues $[44]$, and different cytokine responses $[45,46]$ by comparison to similarly infected BALB/c mice. Although, it is difficult to precisely predict how the reported differences in pathogenesis observed in these two distinct murine backgrounds might be impacted by DbpA and/or DbpB during infection, it is reasonable to suspect that these differences might account for some of the disparity in the results of complementation experiments obtained in the aforementioned studies.

Even though DbpA was required for full infectivity by Bb297 in mice when introduced by needle inoculation (intradermally), neither DbpA, nor DbpB, was required for infection of larval I. scapularis ticks or for transmission of BbKH500 from infected ticks to naïve mice. That DbpA was not required for uptake of the spirochetes by larval ticks was not surprising due to our earlier work demonstrating that DbpA is not expressed by B. burgdorferi harbored within the tick vector [17]. However, based on the data we obtained from needle inoculation of mice with BbKH500, it was surprising that as few as five nymphal ticks could transmit BbKH500. This is especially surprising because semi-quantitative analysis of the total number of spirochetes observed in the midguts of unfed ticks infected with BbKH500 showed a lower spirochete density (approx. 1/field) by comparison to number of bacteria present in the dissected midguts of the Bb297-infected ticks (20-30/field). While these data might suggest a possible defect in either the efficiency of acquisition of the $d b p B A$ mutant or the capacity of this mutant to persist in the tick midgut, there were still sufficient numbers of the mutant spirochetes in these ticks to infect naïve mice. Since it is not known precisely how many spirochetes are transmitted by ticks during the feeding process [47], it was impossible to directly compare the number of $B$. burgdorferi transmitted by tick bite to our needle-challenge experiments. Spirochete numbers within salivary glands during tick feeding have been estimated between 20 [47] and 61 spirochetes [48] per salivary gland pair. These data suggest that an individual tick deposits far fewer than $10^{4}$ spirochetes during the feeding process, and our results have shown that by the needle-inoculation route, $10^{4}$ BbKH500 are not infectious.

In addition to the data presented by Fischer et al. [18], additional reports have attempted to address the role of DbpA, DbpB, and the ECM proteoglycan, decorin, with respect to the infectivity and pathogenesis of $B$. burgdorferi. Brown et al. described B. burgdorferi infection of decorin-deficient mice ( $\left.\mathrm{Dcn}^{-/}\right)$[49]. Brown et al. [49] found that, by comparison to $\mathrm{Dcn}^{+/+}$mice, $\mathrm{Dcn} /-$ mice challenged with a higher dose of $B$. burgdorferi $\left(10^{4}\right)$ had i) fewer infected joints, ii) a reduction in the severity of arthritis, but iii) no significant defect in colonization of the other tissues. Whereas $10^{4}$ wild-type B. burgdorferi were infectious for $\mathrm{Dcn}^{-/}$- mice in the studies by Brown et al. [49], the Bb297 dbpBA-mutant was unable to infect mice at this dose. When considered together, these data support the hypothesis that DbpA, in addition to binding decorin, may have an additional ligand(s) or has another function critical for infectivity. At the present time, the only other known ligand recognized by DbpA is the GAG dermatan sulfate [18], but the contribution of this interaction to the pathogenesis of $B$. burgdorferi remains to be elucidated.

\section{Conclusion}

Despite the disparities between the results of the complementation experiments described in the current study and those obtained by Shi et al. [41], the overall results of our needle-inoculation experiments are in agreement with the most recent conclusion of Shi et al. that mutation of $d b p B A$ results in significant attenuation of $B$. burgdorferi in the murine model of Lyme borreliosis. However, the observation that the $d b p B A$ mutant showed a significant reduction in infectivity when the mice were needle inoculated is overshadowed by the finding that this same mutant was capable of infecting mice via tick challenge. 
Table 3: Oligonucleotide primers used for cloning and PCR confirmation.

\begin{tabular}{ll}
\hline Designation & Sequence \\
\hline kol & GGATCTTAAGAATTTCAAATTTT \\
ko2 & TATAGGCGCGCCAATACTACATGCGACCAATa \\
ko3 & TATAGGCGCGCCTGAAGAGAATCCTCCAACTa \\
ko4 & TTTAGATTCTAAAGTTTAGATAAAAATTGGTCGGG \\
ko5 & AAACAAGTCTTAAAATCACAAGC \\
kan5' & AGCCATATTCAACGGGAAACG \\
kan3' & TTCATATCAGGATTATCAATACC \\
& \\
vlsE-5' & GATGCAGAGAAGGCTGCTGCTGCAGTTAGTGC \\
vlsE-3' & TATAAGCTTTCATCAGAGAGTCTTATTAACAGCAGTCTCAAC \\
BBE22-5' & AAATTAATTTCTTTGATCAACCAAC \\
BBE22-3' & TATATTAAGCTTACTTTGGCTGTCG \\
FlaB5' & ATGATTATCAATCATAATACATCAGCTATTAA \\
FlaB3' & TTATCTAAGCAATGACAAAACATATTGGGGAA \\
compl & \\
comp2 & GGCTTCTCTTTTATTTTTAAGACC \\
comp3 & CATATGTTCCTCCTTCTATTAAATTTAGTTAAATTTAAATTTTAGCCCACb \\
comp4 & AGATCTCATATGATTAAATGTAATAATAAAACTTTb \\
comp5 & GCATGCCTTTGGGTTAATTGCTTTAACc \\
\hline
\end{tabular}

a Ascl site underlined.

b Ndel site underlined.

c Sphl site underlined

The fact that DbpA and DbpB are dispensable for infection via the tick-mediated route of infection suggests that B. burgdorferi transmitted via tick bite are in some way phenotypically different than their in vitro-cultivated counterparts, and/or that tick-derived salivary components, such as Salp15, may assist $B$. burgdorferi during the early infection process [50]. Taken together, these results emphasize the importance of characterizing the impact of a given gene in the infectious lifecycle of $B$. burgdorferi using the natural tick vector, as opposed to using only the artificial needle-challenge model.

\section{Methods}

\section{Bacterial strains and growth conditions}

Infectious, low-passage B. burgdorferi strain 297 (Bb297) [51] was used for these studies. Bacteria were cultivated in vitro in either Barbour-Stoenner-Kelley (BSK)-II [52] or BSK-H Incomplete medium (Sigma-Aldrich, St. Louis, MO) supplemented with 6\% normal rabbit serum (PelFreeze Biologicals, Rogers, AR) at $35^{\circ} \mathrm{C}$ with $5 \% \mathrm{CO}_{2}$. When necessary, BSK media was supplemented with borrelia antibiotic mix (BAM; Sigma-Aldrich), $600 \mu \mathrm{g} / \mathrm{ml}$ kanamycin, or $700 \mu \mathrm{g} / \mathrm{ml}$ streptomycin.

\section{Generation of DbpBA deletion mutant in Bb297}

The dbpBA-deficient Bb297 strain was created by allelic exchange of the $d b p B A$ operon with a kanamycin-resistance cassette, PflgB-kan [53], derived from pBSV2 [54]. The mutagenesis construct, pKHdbpBAko, was created by generating two PCR products that constituted the left and right flanking regions of the $d b p B A$ operon which then were joined via an $A s c I$ restriction site, thus deleting a significant portion of the $d b p B A$ operon. Takara EX Taq polymerase (Takara Bio Inc., Shiga, Japan) and oligonucleotide primers ko1 and ko2 (Table 3) were used to amplify the left arm and ko3 and ko4 (Table 3) were used to amplify the right arm; primers ko2 and ko3 were modified such that $A s c$ I restriction sites would be introduced into the "middle termini" of the two PCR fragments. The resulting PCR products then were digested with AscI and ligated together. The linear ligated product was used as the template in a second PCR amplification containing the primers ko1 and ko4. The resulting PCR product, representing the joined flanking regions, was cloned into pGEM-T Easy vector (Promega Corp., Madison, WI) to generate pGEMT-dbpBA-AscI. The PflgB-kan cassette was from pJD55, a derivative of pJD44 in which the original aph [3']-IIIa was replaced with the PflgB-kan cassette of pBSV2 $[27,54]$. In pJD55, the PflgB-kan cassette is flanked by AscI sites which facilitated the cloning of the marker into the unique AscI site within pGEMT-dbpBA-AscI to create pKHdbpBAko. Primers kan5' and kan3' were used with primers ko1 and ko4 to determine the orientation of the PflgB-kan cassette with respect to the $d b p B A$ operon.

Bb297 were made electrocompetent and transformed with pKHdbpBAko as described by Yang et al [28]. After electroporation with pKHdbpBAko, spirochetes were allowed to recover overnight at $35^{\circ} \mathrm{C}$ without antibiotic selection in $20 \mathrm{ml}$ BSK-H. The cells were diluted in BSK-H 
medium containing the appropriate concentration of antibiotic(s) and aliquoted in 96-well tissue culture plates (Corning, Lowell, MA). Transformants were recovered 721 days after plating from wells in which a red to orange color change of the medium was observed. The presence of viable spirochetes was confirmed visually by dark-field microscopy and clones expanded into BSK medium supplemented with kanamycin.

Transformants were verified as $d b p B A$-deficient mutants by diagnostic PCR using primers ko5 and ko4 (Table 3) followed by analytical restriction enzyme digestion of the PCR product with AscI. DNA for PCR analysis was extracted from borreliae harvested from the expansion cultures. The PCR product generated from amplification of Bb297 DNA (wild-type) is of similar size to that generated by amplification of DNA from a $d b p B A::$ PflgB-kan mutant, but the former lacks $A s c I$ sites. Therefore, to verify that the $d b p B A$ operon was replaced by the PflgB-kan cassette, PCR products from both Bb297 and mutant-derived were digested with AscI prior to agarose-gel electrophoresis. The presence of the $v l_{s E}$ and pncA (BBE22) genes in kanamycin-resistant transformants was confirmed by PCR using vlsE-5' and vlsE-3' for $v l s E$ amplification or BBE22$5^{\prime}$ and BBE22-3' for BBE22 amplification; refer to Table 3 for sequence information. FlaB5' and FlaB3' primers, which amplified the flaB gene of $B$. burgdorferi, were used as a control for DNA integrity. A single $d b p B A$-deficient clone, BbKH500, that retained $v l s E$ and $p n c A$, was chosen for additional PCR-based analyses to compare the endogenous plasmid content of this clone to Bb297. The sequences of the primers utilized for plasmid profiling are provided in Table 4. Nine of the primer pairs have been previously described by Eggers et al. [55]. The remaining primers utilized are unique from those cited by Eggers et al. and were designed primarily based on sequence data from strain 297.

\section{Construction of shuttle plasmids for genetic complementation of BbKH500 with DbpA}

Complementation of DbpA in BbKH500 was achieved by transforming electrocompetent BbKH500 with the $B$. burgdorferi-shuttle plasmid, pKH2000. Because $d b p B$ precedes $d b p A$ in the native operon, it first was necessary to clone the promoter for the $d b p B A$ operon directly in front of the $d b p A$ gene from $B b 297$, thereby removing the $d b p B$ gene. The $d b p B A$ promoter region (Prom) was amplified by PCR using primers comp 1 and comp 2 and the $d b p A$ open reading frame (ORF) was amplified using primer comp3 and comp4. To facilitate fusion of the Prom and $d b p A$ open reading frame fragment, an NdeI restriction site was introduced into the 3 ' end of oligonucleotide comp2 and the $5^{\prime}$ end of primer comp3. Following PCR amplification, the resulting PCR fragments were digested with NdeI and ligated together. A second PCR amplification was performed using the ligation product as the template and the oligonucleotides comp 1 and comp4 for primers. The resultant PCR product was digested with BglII (a unique BglII site is located in the PCR product, $11 \mathrm{bp}$ downstream of the 3' end of the comp1 primer) and SphI (5' end of comp4 primer) then cloned into the BglII and SphI sites of pJD51, a derivative of pJD44 [27], that contains the aadA gene encoding streptomycin resistance in $B$. burgdorferi [56], to create pKH2000. Electroporation of BbKH500 was performed as described above and transformants were selected in the presence of kanamycin and streptomycin. Antibiotic-resistant clones first were checked for the presence of the Prom- $d b p A$ construct by PCR using primers ko5 and comp5 (the latter anneals near the middle of the $d b p A$ coding strand); $\mathrm{Bb} 297=1.2$ $\mathrm{kb}$ product; $\mathrm{BbKH} 500=$ no product; Prom $-d b p A=535 \mathrm{bp}$ product. Next, DbpA expression in clones identified by PCR confirmation was assessed by immunoblot analysis as described below. Clones that expressed DbpA from the Prom- $d b p A$ construct were assessed for the presence of the $v l s E$ and BBE22 genes by PCR amplification using the primers described above. One clone, BbKH501, was chosen for further characterization. PCR-based plasmid profiling was performed on BbKH501, as described above, to compare the plasmid content of this clone to BbKH500 and $\mathrm{Bb} 297$.

\section{Proteinase $K$ digestion of $B$. burgdorferi}

Intact, motile borreliae were exposed to $200 \mu \mathrm{g}$ of proteinase $\mathrm{K}(40 \mathrm{mg} / \mathrm{ml}$; Fisher Scientific, Pittsburgh, PA) or were sham treated for $40 \mathrm{~min}$ at room temperature. To stop the reaction, $10 \mu \mathrm{l}$ of phenylmethylsulfonyl fluoride (50 $\mathrm{mg} / \mathrm{ml}$ in isopropanol; Sigma) was added to each sample and the bacteria prepared for SDS-polyacrylamide gel electrophoresis (PAGE) and immunoblot analysis as described below.

\section{Immunoblot analysis}

$B$. burgdorferi whole-cell lysates were generated by washing the spirochetes with wash buffer (10 mM HEPES, 150 $\mathrm{mM} \mathrm{NaCl}, \mathrm{pH}$ 7.5) three times, incubating the cells in BugBuster plus Benzonase solution (Novagen, Madison, WI) overnight then adding an equal volume of $2 \times$ SDSPAGE running buffer (Bio-Rad Laboratories, Hercules, CA) for a final concentration of $10^{7}$ bacteria $/ \mathrm{ml}$. Wholecell lysates were separated via electrophoresis through $12.5 \%$ SDS-polyacrylamide gels (approximately $10^{7} \mathrm{spi}-$ rochetes per lane) and transferred to nitrocellulose (0.45 $\mu \mathrm{m}$; Bio-Rad Laboratories) for immunoblot analysis. Nitrocellulose membranes were probed with either 6B3DbpA, a mouse monoclonal antibody that specifically recognizes DbpA, chicken anti-B. burgdorferi FlaB IgY, or rat anti-DbpB anti-sera. The monoclonal antibody, 6B3DbpA, was produced in collaboration with the Antibody Production Core facility at UT Southwestern and the pol- 
Table 4: Oligonucleotide primers used for plasmid profiling.

\begin{tabular}{|c|c|c|}
\hline Primer & Sequence & Reference \\
\hline Ip54 5' & ATGAGCAAAAAAGTAATTTTAATAT & [55] \\
\hline 'p54 3' & CACTAATTCTTTTTGAATTACTAAT & [55] \\
\hline cp26 5' & ATGCCTCCAAAAGTGAAGATAAAAA & {$[55]$} \\
\hline cp26 3' & TAGCTTATAATTAAAAATTATTGAT & [55] \\
\hline cp9 5' & ATGCAAAAAATAAACATAGCTAAAT & {$[55]$} \\
\hline cp9 3' & ATCTTCTTCAAGATATTTTAATTATA & {$[55]$} \\
\hline IpI7 5' & GTGTATACTGACCCAAGGTCAATTA & {$[55]$} \\
\hline 'IpI7 3' & CAATAATGTGATATTTTTAAAGAAAT & {$[55]$} \\
\hline Ip25 5' & AAATTAATTTCTTTGATCAACCAAC & This study \\
\hline 'اp25 3' & TATATTAAGCTTACTTTGGCTGTCG & This study \\
\hline 'p28-I 5' & GATGCAGAGAAGGCTGCTGCTGCAGTTAGTGC & This study \\
\hline 'P28-I 3' & TATAAGCTTTCATCAGAGAGTCTTATTAACAGCAGTCTCAAC & This study \\
\hline Ip28-2 5' & ATGGCGCTGATTACATTAATTGTCG & {$[55]$} \\
\hline 'اp28-2 3' & AATCTTGAAGAACCTTGCATCTTTA & {$[55]$} \\
\hline Ip28-3 5' & CTGAAAATGAAGGAGAAGCGGGTGG & [55] \\
\hline 'Ip28-3 3' & TAGGCTAATACCAATTCGTACAAAT & [55] \\
\hline Ip28-4 5' & ATGAAATGCCATATAATTGCAACTA & [55] \\
\hline 'lp28-4 3' & AATCCGACAGATCTGGTTTGTCCAG & {$[55]$} \\
\hline Ip38 5' & ATGATTATTACCCAAACAACGCCC & This study \\
\hline 'اp38 3' & TTTTAAATCCATTTTCACAATATG & This study \\
\hline Ip36 5' & TTCTTATCCCTGACTTTCACTTTTGAGG & This study \\
\hline 'اp36 3' & TCCTTTACTTCTATGTTTTTACTTTCCTTGGT & This study \\
\hline Ip5 5' & ATGAATGGAATAATTAACGATACAC & [55] \\
\hline Ip5 3' & AATATTAGGATGAAGATTATAAATT & {$[55]$} \\
\hline Ip2| 5' & TGTGGTTGCTAAAACCCAAGCGT & This study \\
\hline Ip2| 3' & TTGTTTCTAATTGCTCTGAATTGCATCC & This study \\
\hline Chrom 5' & GATTATCAATCATAATACATCAGC & [55] \\
\hline Chrom 3' & TCTAAGCAATGACAAACATATTGG & {$[55]$} \\
\hline cpl8-I 5' & AGGGGAATGTATTAATTGATAATTCA & This study \\
\hline 'cpl8-I 3' & AGATTTTTTTCAAAACATTTGGCGAT & This study \\
\hline cpl8-2 5' & TCAGAAAGCATACCATTACAAGACAAC & This study \\
\hline cpl8-2 3' & AATAATACCTTTTTCTACGCCCGATA & This study \\
\hline cp32-I 5' & GTTATAATACCTATTCAAGCAGAAAGG & This study \\
\hline cp32-I 3' & GCTCССTTCTAATACTTTTCTATAA & This study \\
\hline cp32-2 5' & CAAGCGAGTTTATTCCCCTTAAA & This study \\
\hline cp32-2 3' & ATTCTAATATTGTCCACTTTATGAAAT & This study \\
\hline cp32-3 5' & ACTTGCAAGAGCACAGGTCTATAATTA & This study \\
\hline ср32-3 3' & CTTAATACAATTAACGTTTCCAGTATA & This study \\
\hline cp32-4 5' & GTATAAATGCTTTTGGTTATAAGCACAC & This study \\
\hline cp32-4 3' & GAAACTCСТTСТСТААССТTTACATAC & This study \\
\hline ср32-5 5' & GCCTTATAAGGAACATAGGTTAAAGG & This study \\
\hline cp32-5 3' & AGATTTCAAGCGCTCCTTCAACAAA & This study \\
\hline cp32-6 5' & GGTGCTTTAGACACAAGAGATGTG & This study \\
\hline cp32-6 3' & GAACAAATTTCAGATTTAACATTTATCG & This study \\
\hline cp32-7 5' & GTCAAATTTAAGCTGTTTTAGCAGTG & This study \\
\hline cp32-7 3' & TATTTACTAАТСТАТTTTTСААТTTTTСА & This study \\
\hline
\end{tabular}

yclonal rat anti-DbpB antisera was described previously [16]. The chicken anti-FlaB antibody was produced in collaboration with Lampire Biological Laboratories (Pipersville, PA). Secondary antibodies were horseradish peroxidase-conjugated goat anti-mouse immunoglobulin G (IgG), donkey anti-chicken IgY, or goat anti-rat IgG (Jackson ImmunoResearch Laboratories, Inc., West Grove, PA) diluted 1:10,000-1:30,000 for chemiluminescent detection. Immunoblots were developed using Immobilon Chemiluminescent Western HRP Substrate
(Millipore, Billerica, MA) and exposed to X-ray film (Phenix Research Products, Hayward, CA).

\section{Infection of mice by needle inoculation}

Prior to use of the cultures in needle-inoculation experiments, the bacterial cell density in each culture was accurately determined by counting spirochetes in no fewer than 60 microscopic fields (400× magnification) using dark-field microscopy. Cultures exhibiting cell aggregation were not used for infections as the presence of clumps 
prevented accurate enumeration of spirochete density. Cultures with spirochetes exhibiting reduced motility also were not used for mouse infections. Three- to five-weekold female $\mathrm{C} 3 \mathrm{H} / \mathrm{HeJ}$ mice (The Jackson Laboratory, Bar Harbor, ME) were used for all studies. Mice were infected via intradermal injection with serial dilutions of $B$. burgdorferi in BSK medium as previously described [16]. At the appropriate time intervals, ear-punch biopsies were harvested using a $2 \mathrm{~mm}$ ear punch, placed in BSK-H medium supplemented with BAM, and cultures were examined by dark-field microscopy for the presence of spirochetes. Aliquots of each culture were passed to media containing antibiotics to confirm the antibiotic-resistance phenotype of the bacteria that were recovered from cultures of the ear punch biopsies. The $\mathrm{ID}_{50}$ was calculated using the method described by Reed and Muench [57]. UT Southwestern is accredited by the International Association for Assessment and Accreditation of Laboratory Animals Care (AAALAC) and all animal protocols were approved by the Institutional Animal Care and Use Committee (IACUC) at UT Southwestern.

\section{Colonization of Ixodes scapularis larvae with B. burgdorferi}

Female $\mathrm{C} 3 \mathrm{H} / \mathrm{HeJ}$ mice were needle inoculated as described above with either Bb297 ( $10^{4}$ spirochetes) or BbKH500 (100 $\mu$ l of a post-exponential growth phase culture; $10^{6}-10^{7}$ spirochetes). Infection of mice was confirmed by ear-punch biopsy culture; 4- and 8-weeks postinoculation for Bb297 and BbKH500, respectively. Naïve, pathogen-free I. scapularis larvae, obtained from the Department of Entomology and Plant Pathology at Oklahoma State University (Stillwater, OK), were allowed to feed to repletion on the infected mice individually housed in cages with raised wire-bottoms above water to facilitate recovery of the ticks. Fed larvae were collected and washed sequentially with $70 \%$ ethanol, deionized water $\left(\mathrm{dH}_{2} \mathrm{O}\right)$, $1 \times$ Fungizone (Gemini Bio-Products, West Sacramento, CA), and $\mathrm{dH}_{2} \mathrm{O}$ before placing them in $100 \%$ cotton fabric-lined Petri dish molting chambers for storage until they molted to the nymphal stage. The molting chambers containing the ticks were housed in a humidified chamber (97-98\% humidity) containing saturated potassium sulfate solution at $20^{\circ} \mathrm{C}$ with a $16 \mathrm{~h}$ light, $8 \mathrm{~h}$ dark cycle. Unfed nymphs were collected and stored in autoclaved glass vials containing approximately $1 \mathrm{~cm}$ of sand. The vials were closed with vented lids and the ticks housed as described above.

\section{Direct immunofluorescence assay (IFA) on B. burgdorferi- infected ticks}

Prior to placement of unfed (flat) nymphs on naïve mice, five nymphs from each of two individual mice were dissected on silylated slides (CEL Associates, Inc., Pearland, TX) in $50 \mu \mathrm{l}$ phosphate-buffered saline containing $10 \mathrm{mM}$
$\mathrm{MgCl}_{2}$. At the time testing was performed, approximately 11 weeks had elapsed since these ticks had fed to repletion as larvae. Midgut tissues were extracted from the ticks, allowed to dry on the slides, blocked with Tris-buffered saline with $0.1 \%$ Tween-20, then probed with a FITC-conjugated rabbit anti-B. burgdorferi antibody (Fitzgerald Industries International, Inc., Concord, MA) as described previously [27].

\section{Transmission of $B$. burgdorferi from infected nymphal ticks to naïve mice}

To assess the transmissibility of strain Bb297 and BbKH500 from flat nymphs to naïve mice, either five or ten ticks were allowed to feed to repletion on three- to five-week-old female $\mathrm{C} 3 \mathrm{H} / \mathrm{HeJ}$ mice. At three-weeks postinfestation, ear-punch biopsies were harvested from each mouse and cultured in BSK-H medium without antibiotics to determine infection status. Borreliae from the earpunch biopsy cultures were transferred to BSK-H medium supplemented with kanamycin and streptomycin to confirm their antibiotic-resistance phenotypes.

\section{Statistical analysis}

Statistical analysis was performed with assistance from the UT Southwestern Clinical Sciences Department. Both chisquare and Fisher's exact test were applied to the mouse infection data in pair-wise comparisons between experimental groups.

\section{Authors' contributions}

JSB and KEH performed experiments and analyzed results. JSB, KEH, and MVN participated in experimental designs and co-wrote the manuscript. All authors read and approved the manuscript.

\section{Acknowledgements}

We thank Sarah Sutton, Carl Stephens, and Rachel Metz for technical assistance, and Alan Elliot for assistance with statistical analysis. We also thank Melissa Caimano and Justin Radolf for strain 297-specific primer sequences for plasmid profiling experiments. This work was supported by Public Health Service Grant Al-05 332 and Al-059602. J.S.B. was supported by the National Institutes of Health Training Grant T32-A107520 and the Ruth L. Kirschstein National Research Service Award F32-A1058487 from the National Institutes of Health.

\section{References}

I. Steere AC, Coburn J, Glickstein L: The emergence of Lyme disease. J Clin Invest 2004, I I 3(8): I093-I I0I.

2. Coburn J, Fischer JR, Leong JM: Solving a sticky problem: new genetic approaches to host cell adhesion by the Lyme disease spirochete. Mol Microbiol 2005, 57(5): I I82-I I 95.

3. Coburn J, Medrano M, Cugini C: Borrelia burgdorferi and its tropisms for adhesion molecules in the joint. Curr Opin Rheumatol 2002, I 4(4):394-398.

4. Zambrano MC, Beklemisheva AA, Bryksin AV, Newman SA, Cabello FC: Borrelia burgdorferi binds to, invades, and colonizes native type I collagen lattices. Infect Immun 2004, 72(6):3|38-3|46.

5. Finlay BB, Falkow S: Common themes in microbial pathogenicity revisited. BMC Microbiol 1997, 6 I: I 36-169. 
6. Fischer JR, LeBlanc KT, Leong JM: Fibronectin binding protein BBK32 of the Lyme disease spirochete promotes bacteria attachment to glycosaminoglycans. Infect Immun 2006, 74(I):435-44I.

7. Grab DJ, Givens C, Kennedy R: Fibronectin-binding activity in Borrelia burgdorferi. Biochim Biophys Acta I998, I 407(2): I 35- I 45.

8. Behera AK, Durand E, Cugini C, Antonara S, Bourassa L, Hildebrand E, Hu LT, Coburn J: Borrelia burgdorferi BBB07 interaction with integrin alpha3betal stimulates production of proinflammatory mediators in primary human chondrocytes. Cell Microbiol 2007/09/08 edition. 2008, I0(2):320-33I.

9. Coburn J, Chege W, Magoun L, Bodary SC, Leong JM: Characterization of a candidate Borrelia burgdorferi beta3-chain integrin ligand identified using a phage display library. Mol Microbiol 1999, 34:926-940.

10. Guo BP, Norris SJ, Rosenberg LC, Hook M: Adherence of Borrelia burgdorferi to the proteoglycan decorin. Infect Immun 1995 , 63:3467-3472.

II. Isaacs RD: Borrelia burgdorferi bind to epithelial cell proteoglycans. J Clin Invest 1994, 93(2):809-8I9.

12. Parveen N, Leong JM: Identification of a candidate glycosaminoglycan-binding adhesin of the Lyme disease spirochete Borrelia burgdorferi. Mol Microbiol 2000, 35(5):1220-1234.

13. Probert WS, Johnson BJ: Identification of a $\mathbf{4 7} \mathbf{~ k D a}$ fibronectinbinding protein expressed by Borrelia burgdorferi isolate B3 I. Mol Microbiol 1998, 30:1003-1015.

14. Coburn J, Cugini C: Targeted mutation of the outer membrane protein P66 disrupts attachment of the Lyme disease agent, Borrelia burgdorferi, to integrin alphavbeta3. Proc Natl Acad Sci USA 2003, I00(1 2):730I-7306.

15. Guo BP, Brown EL, Dorward DW, Rosenberg LC, Hook M: Decorin-binding adhesins from Borrelia burgdorferi. Mol Microbiol 1998, 30:7II-723

16. Hagman KE, Lahdenne P, Popova TG, Porcella SF, Akins DR, Radolf JD, Norgard MV: Decorin-binding protein of Borrelia burgdorferi is encoded within a two-gene operon and is protective in the murine model of Lyme borreliosis. Infect Immun 1998, 66:2674-2683

17. Hagman KE, Yang X, Wikel SK, Schoeler GB, Caimano MJ, Radolf JD, Norgard MV: Decorin-binding protein A (DbpA) of Borrelia burgdorferi is not protective when immunized mice are challenged via tick infestation and correlates with the lack of DbpA expression by B. burgdorferi in ticks. Infect Immun 2000 , 68:4759-4764

18. Fischer JR, Parveen N, Magoun L, Leong JM: Decorin-binding proteins $A$ and $B$ confer distinct mammalian cell type-specific attachment by Borrelia burgdorferi, the Lyme disease spirochete. Proc Natl Acad Sci USA 2003, I 00( I 2):7307-73I2.

19. Roberts WC, Mullikin BA, Lathigra R, Hanson MS: Molecular analysis of sequence heterogeneity among genes encoding decorin binding proteins $A$ and $B$ of Borrelia burgdorferi sensu lato. Infect Immun 1998, 66:5275-5285.

20. Hodzic E, Feng S, Freet KJ, Borjesson DL, Barthold SW: Borrelia burgdorferi population kinetics and selected gene expression at the host-vector interface. Infect Immun 2002, 70(7):3382-3388.

2I. Schulte-Spechtel U, Lehnert G, LiegI G, Fingerle V, Heimerl C, Johnson B], Wilske B: Significant improvement of the recombinant Borrelia-specific immunoglobulin $G$ immunoblot test by addition of VIsE and a DbpA homologue derived from Borrelia garinii for diagnosis of early neuroborreliosis. J Clin Microbiol 2003, 4 I (3): I299-1303.

22. Nowalk AJ, Gilmore RD Jr., Carroll JA: Serologic proteome analysis of Borrelia burgdorferi membrane-associated proteins. Infect Immun 2006, 74(7):3864-3873.

23. Shi $Y, X u$ Q, Seemanapalli SV, McShan K, Liang FT: The dbpBA locus of Borrelia burgdorferi is not essential for infection of mice. Infect Immun 2006, 74(I I):6509-65I 2.

24. Xu Y, Johnson RC: Analysis and comparison of plasmid profiles of Borrelia burgdorferi sensu lato strains. J Clin Microbiol 1995 , 33(I0):2679-2685.

25. Caimano MJ, Eggers CH, Hazlett KR, Radolf JD: RpoS is not centra to the general stress response in Borrelia burgdorferi but does control expression of one or more essential virulence determinants. Infect Immun 2004/10/27 edition. 2004, 72(II):6433-6445.
26. Hubner A, Revel AT, Nolen DM, Hagman KE, Norgard MV: Expression of a luxS gene is not required for Borrelia burgdorferi infection of mice via needle inoculation. Infect Immun 2003, 7 I (5):2892-2896

27. Revel AT, Blevins JS, Almazan C, Neil L, Kocan KM, de la Fuente J, Hagman KE, Norgard MV: bptA (bbe I6) is essential for the persistence of the Lyme disease spirochete, Borrelia burgdorferi, in its natural tick vector. Proc Natl Acad Sci USA 2005, I 02(19):6972-6977.

28. Yang XF, Pal U, Alani SM, Fikrig E, Norgard MV: Essential role for OspA/B in the life cycle of the Lyme disease spirochete. J Exp Med 2004, 199(5):641-648.

29. Lawrenz MB, Wooten RM, Norris SJ: Effects of vlsE complementation on the infectivity of Borrelia burgdorferi lacking the linear plasmid Ip28-I. Infect Immun 2004, 72(I I):6577-6585.

30. Purser JE, Lawrenz MB, Caimano MJ, Howell JK, Radolf JD, Norris SJ: A plasmid-encoded nicotinamidase (PncA) is essential for infectivity of Borrelia burgdorferi in a mammalian host. Mol Microbiol 2003, 48(3):753-764.

31. Brown EL, Guo BP, O'Neal P, Hook M: Adherence of Borrelia burgdorferi. Identification of critical lysine residues in DbpA required for decorin binding. J Biol Chem 1999/09/03 edition. 1999, 274(37):26272-26278.

32. Pikas DS, Brown EL, Gurusiddappa S, Lee LY, Xu Y, Hook M: Decorin-binding sites in the adhesin DbpA from Borrelia burgdorferi: a synthetic peptide approach. I Biol Chem 2003/05/23 edition. 2003, 278(33):30920-30926.

33. Hanson MS, Cassatt DR, Guo BP, Patel NK, McCarthy MP, Dorward DW, Hook M: Active and passive immunity against Borrelia burgdorferi decorin binding protein $A$ (DbpA) protects against infection. Infect Immun 1998/05/09 edition. 1998, 66(5):2143-2I53

34. Coburn J: Adhesion mechanisms of the Lyme disease spirochete, Borrelia burgdorferi. Curr Drug Targets Infect Disord 200I, I(2): $17|-| 79$

35. Leong JM, Morrissey PE, Ortega-Barria E, Pereira ME, Coburn J: Hemagglutination and proteoglycan binding by the Lyme disease spirochete, Borrelia burgdorferi. Infect Immun 1995, 63(3):874-883

36. Parveen N, Cornell KA, Bono JL, Chamberland C, Rosa P, Leong JM: Bgp, a secreted glycosaminoglycan-binding protein of Borrelia burgdorferi strain N40, displays nucleosidase activity and is not essential for infection of immunodeficient mice. Infect Immun 2006, 74(5):3016-3020.

37. Li X, Liu X, Beck DS, Kantor FS, Fikrig E: Borrelia burgdorferi lacking BBK32, a fibronectin-binding protein, retains full pathogenicity. Infect Immun 2006, 74(6):3305-33।3.

38. Coburn J, Leong JM, Erban JK: Integrin alpha Ilb beta 3 mediates binding of the Lyme disease agent Borrelia burgdorferi to human platelets. Proc Natl Acad Sci USA 1993, 90(I 5):7059-7063.

39. Sadziene A, Thomas DD, Barbour AG: Borrelia burgdorferi mutant lacking Osp: biological and immunological characterization. Infect Immun 1995, 63(4): I573-1580.

40. Kawabata $\mathrm{H}$, Norris SJ, Watanabe H: BBE02 disruption mutants of Borrelia burgdorferi B3I have a highly transformable, infectious phenotype. Infect Immun 2004, 72(12):7147-7I54.

4I. Shi Y, Xu Q, McShan K, Liang FT: Both decorin-binding proteins $A$ and $B$ are critical for the overall virulence of Borrelia burgdorferi. Infect Immun 2008, 76(3): I239-1246.

42. Barthold SW, Beck DS, Hansen GM, Terwilliger GA, Moody KD: Lyme borreliosis in selected strains and ages of laboratory mice. J Infect Dis 1990, 162(I):133-138.

43. Barthold SW, Persing DH, Armstrong AL, Peeples RA: Kinetics of Borrelia burgdorferi dissemination and evolution of disease after intradermal inoculation of mice. Am J Pathol I99|, I39(2):263-273.

44. Yang L, Weis JH, Eichwald E, Kolbert CP, Persing DH, Weis Jl: Heritable susceptibility to severe Borrelia burgdorferi-induced arthritis is dominant and is associated with persistence of large numbers of spirochetes in tissues. Infect Immun 1994, 62(2):492-500

45. Keane-Myers A, Nickell SP: Role of IL-4 and IFN-gamma in modulation of immunity to Borrelia burgdorferi in mice. J Immunol 1995, I 55(4):2020-2028.

46. Zeidner N, Mbow ML, Dolan M, Massung R, Baca E, Piesman J: Effects of Ixodes scapularis and Borrelia burgdorferi on mod- 
ulation of the host immune response: induction of a TH2 cytokine response in Lyme disease-susceptible $(\mathrm{C} 3 \mathrm{H} / \mathrm{HeJ})$ mice but not in disease-resistant (BALB/c) mice. Infect Immun 1997, 65(8):3100-3106.

47. Piesman J, Schneider BS, Zeidner NS: Use of quantitative PCR to measure density of Borrelia burgdorferi in the midgut and salivary glands of feeding tick vectors. Journal of Clinical Microbiology 200I, 39:4I45-4I48.

48. Ohnishi J, Piesman J, de Silva AM: Antigenic and genetic heterogeneity of Borrelia burgdorferi populations transmitted by ticks. Proc Natl Acad Sci USA 200I, 98:670-675.

49. Brown EL, Wooten RM, Johnson BJ, lozzo RV, Smith A, Dolan MC Guo BP, Weis JJ, Höök M: Resistance to Lyme disease in decorin-deficient mice. J Clin Invest 200I, I 07:845-852.

50. Ramamoorthi N, Narasimhan S, Pal U, Bao F, Yang XF, Fish D, Anguita J, Norgard MV, Kantor FS, Anderson JF, Koski RA, Fikrig E: The Lyme disease agent exploits a tick protein to infect the mammalian host. Nature 2005, 436(7050):573-577.

51. Norton Hughes CA, Kodner CB, Johnson RC: DNA analysis of Borrelia burgdorferi NCH-I, the first northcentral U.S. human Lyme disease isolate. Journal of Clinical Microbiology 1992, 30:698-703.

52. Pollack RJ, Telford SR, Spielman A: Standardization of medium for culturing Lyme disease spirochetes. J Clin Microbiol 1993, 3 I(5): I25I-I255.

53. Bono JL, Elias AF, Kupko JJ 3rd, Stevenson B, Tilly K, Rosa P: Efficient targeted mutagenesis in Borrelia burgdorferi. J Bacteriol 2000/ 04/13 edition. 2000, I 82(9):2445-2452.

54. Stewart PE, Thalken R, Bono JL, Rosa P: Isolation of a circular plasmid region sufficient for autonomous replication and transformation of infectious Borrelia burgdorferi. Mol Microbiol 200I/02/I 3 edition. 200I, 39(3):7I4-72I.

55. Eggers CH, Caimano MJ, Clawson ML, Miller WG, Samuels DS, Radolf JD: Identification of loci critical for replication and compatibility of a Borrelia burgdorferi cp32 plasmid and use of a cp32-based shuttle vector for the expression of fluorescent reporters in the lyme disease spirochaete. Mol Microbiol 2002/ 05/03 edition. 2002, 43(2):28I-295.

56. Frank KL, Bundle SF, Kresge ME, Eggers CH, Samuels DS: aadA confers streptomycin resistance in Borrelia burgdorferi. J Bacteriol 2003/I I/05 edition. 2003, 185(22):6723-6727.

57. Reed LJ, Muench $\mathrm{H}$ : A simple method of estimating fifty percent endpoints. Am J Hyg 1938, 27:493-497.

Publish with Bio Med Central and every scientist can read your work free of charge

"BioMed Central will be the most significant development for disseminating the results of biomedical research in our lifetime. "

Sir Paul Nurse, Cancer Research UK

Your research papers will be:

- available free of charge to the entire biomedical community

- peer reviewed and published immediately upon acceptance

- cited in PubMed and archived on PubMed Central

- yours - you keep the copyright
BioMedcentral 